\title{
Effect of freeze-drying and gamma irradiation on biomechanical properties of bovine pericardium
}

\begin{abstract}
Freeze-drying and gamma irradiation are the techniques widely use in tissue banking for preservation and sterilization of tissue grafts respectively. However, the effect of these techniques on biomechanical properties of bovine pericardium is poorly known. A total of 300 strips of bovine pericardium each measured $4 \mathrm{~cm}-1 \mathrm{~cm}$ were used in this study to evaluate the effect of freeze-drying on biomechanical properties of fresh bovine pericardium and the effect of gamma irradiation on biomechanical properties of freeze-dried bovine pericardium. The strips were divided into three equal groups, which consist of 100 strips each group. The three groups were fresh bovine pericardium, freeze-dried bovine pericardium and irradiated freeze-dried bovine pericardium. The biomechanical properties of the pericardial strips were measured by a computer controlled instron tensiometer while the strips thickness was measured by Mitutoyo thickness gauge. The results of the study revealed that freezedrying has no significant $(\mathrm{p}>0.05)$ effect on the tensile strength, Young's modulus (stiffness) and elongation rate of fresh bovine pericardium. Irradiation with $25 \mathrm{kGy}$ gamma rays caused significant decreased in the tensile strength, Young's modulus and elongation rate of the freeze-dried pericardium. However, gamma irradiation has no significant effect on the thickness of freeze-dried bovine pericardium, while freeze-drying caused significant decreased in the thickness of the fresh bovine pericardium. The outcome of this study demonstrated that freeze-drying has no significant effect on the biomechanical properties of fresh bovine pericardium, and gamma irradiation caused significant effect on the biomechanical properties of freezedried bovine pericardium.
\end{abstract}

Keyword: Biomechanical properties, Freeze-dried bovine pericardium, Gamma irradiation 